\title{
Chiral ${ }^{1} \mathrm{H}$ NMR of Atropisomeric Quinazolinones With Enantiopure Phosphoric Acids
}

\author{
Chaofei Wu, Hongxin Liu*, Juan Li, Hong-Ping Xiao, Xinhua Li and Jun Jiang* \\ College of Chemistry and Materials Engineering, Wenzhou University, Wenzhou, China
}

A chiral phosphoric acid promoted enantioselective NMR analysis of atropisomeric quinazolinones was described, in which a variety of racemic arylquinazolinones such as afloqualone and IC-87114 were well recognized with up to $0.21 \mathrm{ppm} \Delta \Delta \delta$ value. With this method, the optical purities of different non-racemic substrates can be fast evaluated with high accuracy.

Keywords: chiral recognition, ${ }^{1} \mathrm{H}$ NMR analysis, quinazolinones, chiral phosphoric acid, high accuracy

OPEN ACCESS

Edited by:

Ramon Rios,

University of Southampton,

United Kingdom

Reviewed by:

Andrea Mazzanti,

Università degli Studi di Bologna, Italy

Thomas Poisson,

Institut National des Sciences

Appliquées de Rouen, France

*Correspondence:

Hongxin Liu

hongxin-107@163.com

Jun Jiang

junjiang@wzu.edu.cn

Specialty section:

This article was submitted to

Organic Chemistry,

a section of the journal

Frontiers in Chemistry

Received: 07 May 2018

Accepted: 29 June 2018

Published: 17 August 2018

Citation:

Wu C, Liu H, Li J, Xiao H-P, Li X and Jiang J (2018) Chiral ${ }^{1}$ H NMR of

Atropisomeric Quinazolinones With Enantiopure Phosphoric Acids.

Front. Chem. 6:300.

doi: 10.3389/fchem.2018.00300
After the first experimental detection of atropisomerism by Christie and Kenner in 1922 (Christie and Kenner, 1922), axial chirality was gradually recognized as an important type of molecular asymmetry which derived from the restricted rotation of a single bond in biaryls, amines, etc. For example, axially chiral BINAP and its analogs were found to be excellent ligands in various asymmetric catalytic transformations (Miyashita et al., 1980; Akutagawa, 1995; Kumobayashi et al., 2001; Brunel, 2005, 2007; Genet et al., 2014), while a lot of optically active biaryl natural products were successfully isolated and identified in the past few decades (Bringmann et al., 2011; Smyth et al., 2015). Besides, atropisomers were found to exhibit different pharmacodynamics and pharmacokinetics in many cases (Eichelbaum and Gross, 1996; Clayden et al., 2009). Thus, exploring efficient chiral recognition and determination method for atropisomeric compounds is crucial to the asymmetric synthesis as well as structure-bioactivity study. With the fast development of analysis technology, GC (Schurig and Nowotny, 1990), IR (Reetz et al., 1998), HPLC (Han, 1997), circular dichroism (Ding et al., 1999; Nieto et al., 2008, 2010; Ghosn and Wolf, 2009), fluorescence spectroscopy (James et al., 1995; Mei and Wolf, 2004; Pu, 2004; Zhao et al., 2004; Li et al., 2005; Tumambac and Wolf, 2005; Liu et al., 2009), electrophoresis technologies (Reetz et al., 2000) and NMR spectroscopy have been frequently employed in chiral determinations. Among these methods, we are particularly interested in the NMR based chiral analysis method which employs chiral shift reagents (CSRs) (Frazer et al., 1971; Goering et al., 1971; Yeh et al., 1986; Ghosh et al., 2004; Yang et al., 2005) or chiral solvating reagents (CSAs) (Pirkle, 1966; Lancelot et al., 1969; Parker, 1991; Wenzel and Wilcox, 2003; Seco et al., 2004; Lovely and Wenzel, 2006; Ema et al., 2007; Wenzel, 2007; Iwaniuk and Wolf, 2010; Moon et al., 2010; Gualandi et al., 2011; Pham and Wenzel, 2011; Quinn et al., 2011; Wenzel and Chisholm, 2011; Ma et al., 2012; Labuta et al., 2013; Zhou et al., 2015; Akdeniz et al., 2016; Bian et al., 2016a,b; Huang et al., 2016) to directly differentiate enantiomers of the analytes, since it takes many advantages such as easy operation, fast evaluation, broad analyte scope and so on. In 2017, we reported a chiral phosphoric acid (CPA) promoted enantioselective NMR analysis of indoloquinazoline alkaloid type tertiary alcohols with high efficiency and wide application; this methodology was also employed in the fast optimization of reaction conditions in amino acid metal salt catalyzed asymmetric aldol reaction via direct analysis of the reaction mixture without purification (Liu et al., 2017). Inspired by this result and given the growing interest in axially chiral compounds, 


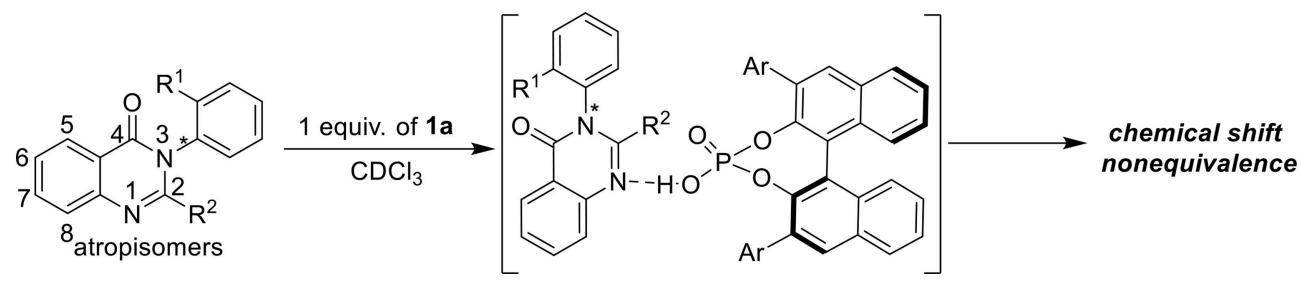

FIGURE 1 | Chiral ${ }^{1} \mathrm{H}$ NMR analysis of arylquinazolinones with a chiral phosphoric acid.

TABLE 1 | Evaluating the chiral recognition abilities of chiral phosphoric acids $\mathbf{1}$ with $\mathbf{2 a}$

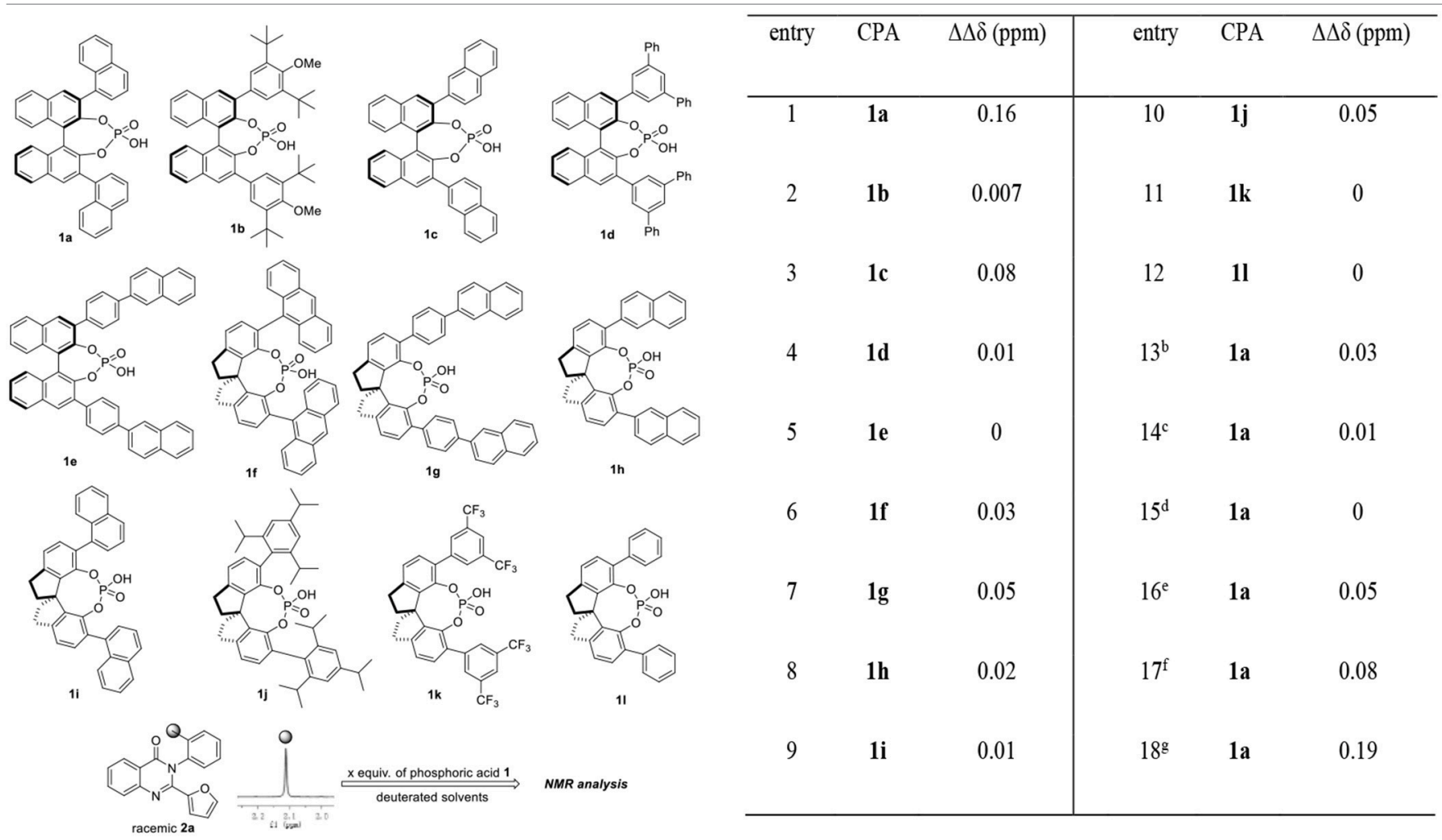

a All samples were prepared by mixing $1(0.01 \mathrm{mmol})$ and the guests $2 \mathrm{a}(0.01 \mathrm{mmol})$ in $\mathrm{CDCl}_{3}$ at $25^{\circ} \mathrm{C}$.

${ }^{b}$ Acetone- $D_{6}$ was used as deuterated solvent.

${ }^{c} \mathrm{CD}_{3} \mathrm{OD}$ was used as deuterated solvent.

${ }^{d}$ DMSO- $D_{6}$ was used as deuterated solvent.

e 0.2 equiv. of 1 a was used.

${ }^{f} 0.5$ equiv. of 1 a was used.

g1.5 equiv. of 1 a was used.

atropisomeric arylquinazolinones, which are constituents of various biologically active natural products and pharmaceutical compounds, were chosen as the next target to further evaluate the chiral recognition ability of chiral phosphoric acids (Figure 1). Herein, we wish to report our preliminary results on this topic: in the presence of $0.2-1.5$ equiv. of $\alpha$-naphthyl phosphoric acid, a variety of racemic arylquinazolinones including afloqualone and IC-87114 were well recognized with up to $0.21 \mathrm{ppm} \Delta \Delta \delta$ value; additionally, the corresponding analysis system can also be employed in the accurate determination of enantioselectivities of chiral arylquinazolinones.
Our exploration of this hypothesis began with evaluating the interaction between chiral phosphoric acids 1 and racemic 2-(furan-2-yl)-3-(o-tolyl)quinazolin-4(3H)-one $2 \mathrm{a}$ in $\mathrm{CDCl}_{3}$ at $25^{\circ} \mathrm{C}$. As shown in Table 1 , after additions of 1 equiv. of chiral phosphoric acids to racemic $2 \mathrm{a}$, chemical shift non-equivalences were observed. However, the structure of phosphoric acids has obvious influence on the recognition result. For example, BINOL derived phosphoric acid 1a was found to be the best host which afforded a baseline resolution and the largest chemical shift nonequivalence of methyl $\mathrm{H}$ signal of $2 \mathrm{a}(0.16 \mathrm{ppm})$, while spiro-phosphoric acid $1 \mathrm{i}$ with the same substituents exhibited 
TABLE 2 | Differentiating the enantiomers of different racemic 3-arylquinazo-linones derivatives $\mathbf{2}$ in the presence of phosphoric acid $\mathbf{1 a}$ a

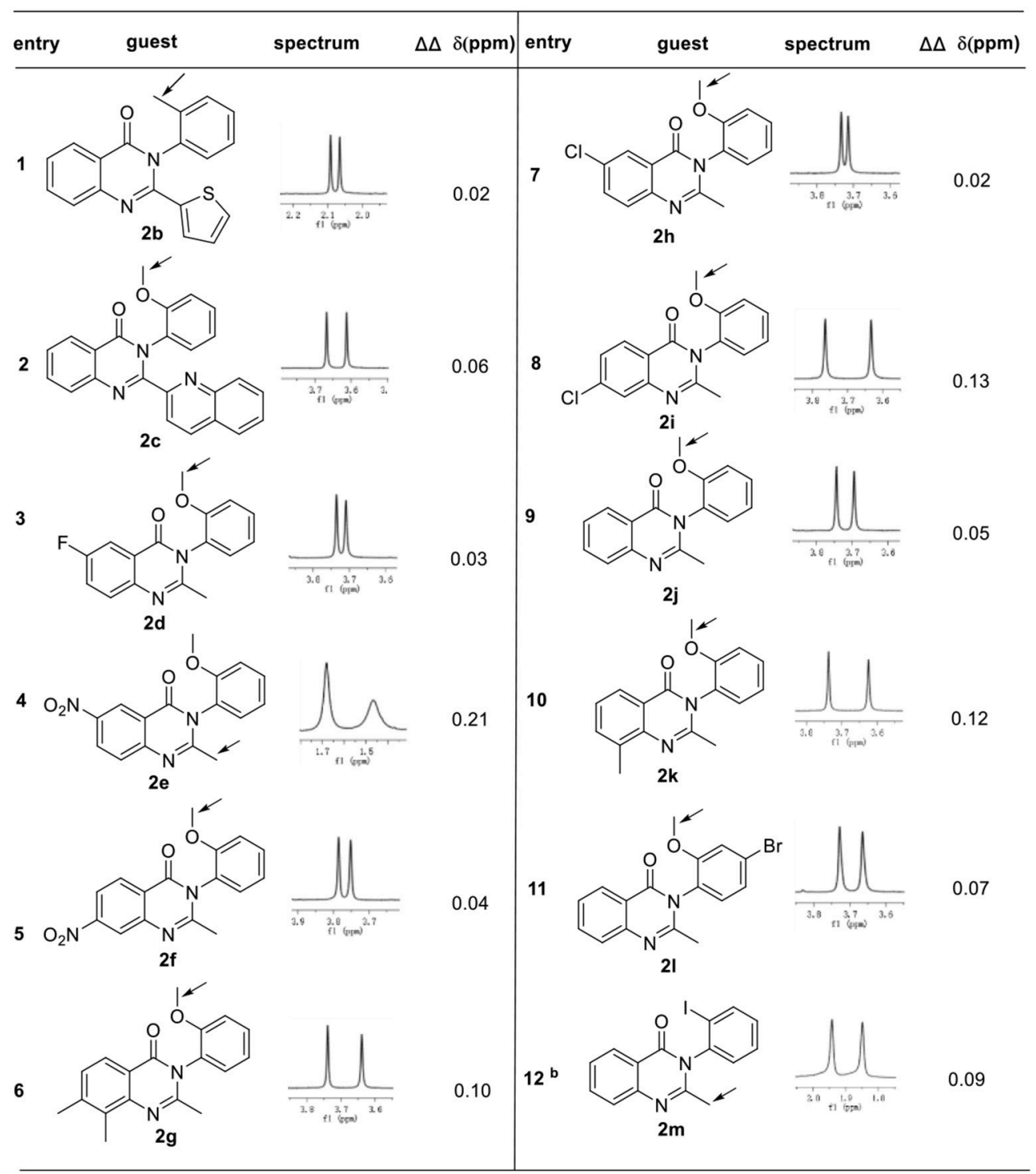

a All samples were prepared by mixing $\mathbf{1 a}(0.01 \mathrm{mmol})$ and the guests $2(0.01 \mathrm{mmol})$ in $\mathrm{CDCl}_{3}$ at $25^{\circ} \mathrm{C}$

b $0.015 \mathrm{mmol} 1 \mathrm{a}$ was used.<smiles>Nc1ccc2nc(CF)n(-c3c(O)cccc3CF)c(=O)c2c1</smiles>

afloqualone

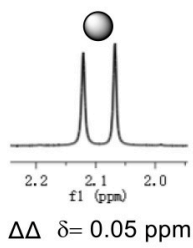

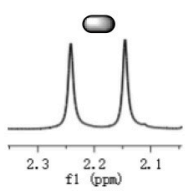

$\Delta \Delta \delta=0.09 \mathrm{ppm}$

FIGURE 2 | afloqualone and IC-87114 were discriminated under standard conditions. 


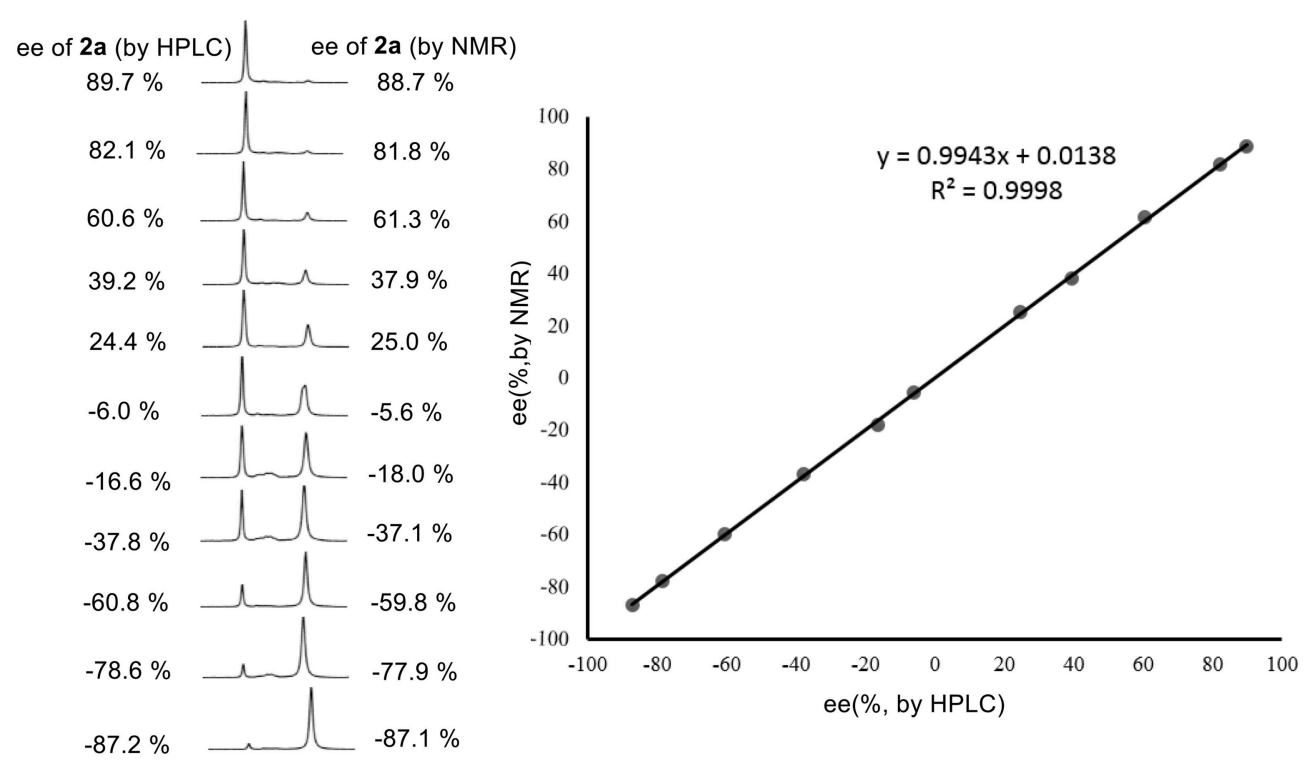

FIGURE $3 \mid{ }^{1} \mathrm{H}$ NMR signals of non-racemic $\mathbf{2 a}$ samples in the presence of 1 equiv. of $\mathbf{1 a}$ in $\mathrm{CDCl}_{3}$ (left); linear relationship between NMR measured ee values versus the HPLC determined ee values (right).

poor chiral recognition ability; additionally, phosphoric acids with very bulky substituents such as $1 \mathrm{~b}$ and $1 \mathrm{j}$ also failed to provide satisfactory discriminating results. (For details, see Supplementary Materials). With optimal host 1a in hand, the effect of deuterated solvents was also studied. It was shown that the interaction between chiral phosphoric acid $1 \mathrm{a}$ and guest $2 \mathrm{a}$ existed even in protic and polar solvents $\left(\mathrm{CD}_{3} \mathrm{OD}\right.$, acetone- $\mathrm{D}_{6}$, DMSO-D 6 ); however, $\mathrm{CDCl}_{3}$ was still found to be the best choice of solvent. To further explore the chiral recognition ability of $1 \mathrm{a}$, attempts to evaluate the influence of the amount of $1 \mathrm{a}$ were also carried out. Generally, larger amount of 1a led to better recognition results (entries 1, 16-18). Noticeably, it was found that $20 \mathrm{~mol} \%$ la was enough to give clear baseline resolution of $2 \mathrm{a}$ under standard analysis condition, albeit with smaller chemical shift nonequivalence (entry 16, $0.05 \mathrm{ppm}$ ). Finally, as the optimal compromise between discriminating result and atom economy, 1 equiv. of chiral phosphoric acid 1a was chosen in standard analysis conditions.

The general applicability of these conditions for a variety of racemic 3-arylquinazolinones was fully demonstrated in Table 2. In the presence of 1 equiv. of phosphoric acid $1 \mathrm{a}$ in $0.5 \mathrm{~mL}$ $\mathrm{CDCl}_{3}$ at $25^{\circ} \mathrm{C}$, a number of racemic 3-arylquinazolinones derivatives $\mathbf{2} \mathbf{b}-\mathbf{2} \mathbf{m}$ with various substituents can be well resolved. For example, other aromatic moieties such as thiophene or quinoline on 2 position of 3-arylquinazolinones can also result in good enantiodiscrimination, albeit with smaller $\Delta \Delta \delta$ value (2b and 2c, 0.02-0.06 ppm); besides, 2-methyl substituted 3arylquinazolinones with either electron-withdrawing group or electron-donating group on $6,7,8$ position were proved to be good guests under optimal conditions, affording baseline resolutions with good chemical shift nonequivalence $(\mathbf{2 d - 2 k}$, 0.02-0.21 ppm). Noticeably, 3-(2-methoxyphenyl)-2-methyl6-nitroquinazolin-4(3H)-one $2 \mathrm{e}$ was well recognized with the largest $\Delta \Delta \delta$ value of $0.21 \mathrm{ppm}$. Additionally, different substituents such as methyl, methoxyl or halogen group on the $\mathrm{N}-3$ benzene ring were also well tolerated. However, when $2^{\prime}$ iodo-benzene was used as substituents on N-3 position, 1.5 equiv of 1a was found necessary to afford satisfactory result (entry 12).

To demonstrate the practical utility of our methodology, commercial drugs (candidate) were next selected for chiral recognition with phosphoric acid 1a. To our delight, racemic 3-arylquinazolinone type bioactive molecules such as multifunctional afloqualone and IC-87114 can be well enantiodiscriminated with baseline resolutions under standard analysis conditions (Figure 2), for details, see

\section{Supplementary Materials.}

Encouraged by these good discrimination results, this methodology was subsequently applied to the enantiomeric determination of various non-racemic 2a samples. As shown in Figure 3, the optical purities of $\mathbf{2 a}$ can be accurately obtained by integrating the corresponding $\mathrm{H}$ signals of methyl group of $2 \mathbf{a}$ in the presence of 1 equiv. of 1a. Compared with those data obtained from chiral HPLC analysis, excellent linear relationship and up to $1.4 \%$ absolute errors were obtained.

\section{CONCLUSIONS}

In conclusion, we developed an efficient chiral NMR analysis method for atropisomeric quinazolinones, in which chiral phosphoric acid shows excellent abilities to discriminate the enantiomers of various 3-arylquinazolinones with good chemical shift non-equivalence. With this method, the optical purities of different non-racemic 2a can be fast evaluated with high accuracy. Further studies on the interactions of chiral phosphoric acid with other analytes are currently underway. 


\section{AUTHOR CONTRIBUTIONS}

JJ and HL designed the project, guided the study, and polished the manuscript. CW and JL: conducted the experiments and characterized the samples. H-PX and XL revised the manuscript.

\section{ACKNOWLEDGMENTS}

This work was supported by National Natural Science Foundation of China (21472141 and 21571144), Zhejiang

\section{REFERENCES}

Akdeniz, A., Minami, T., Watanabe, S., Yokoyama, M., and Ema, T. (2016). Determination of enantiomeric excess of carboxylates by fluorescent macrocyclic sensors. Chem. Sci. 7, 2016-2022. doi: 10.1039/C5SC04235F

Akutagawa, S. (1995). Asymmetric synthesis by metal BINAP catalysts. Appl. Catal. A Gen., 128, 171-207. doi: 10.1016/0926-860X(95)00097-6

Bian, G., Yang, S., Huang, H., Zong, H., and Song, L. (2016a). A bisthiourea-based $1 \mathrm{H}$ NMR chiral sensor for chiral discrimination of a variety of chiral compounds. Sens. Actuators B Chem. 231, 129-134. doi: 10.1016/j.snb.2016.03.002

Bian, G., Yang, S., Huang, H., Zong, H., Song, L., Fan, H., et al. (2016b). Chirality sensing of tertiary alcohols by a novel strong hydrogen-bonding donor-selenourea. Chem. Sci. 7, 932-938. doi: 10.1039/C5SC03780H

Bringmann, G., Gulder, T., Gulder, T. A., and Breuning, M. (2011). Atroposelective total synthesis of axially chiral biaryl natural products. Chem. Rev. 111, 563-639. doi: 10.1021/cr100155e

Brunel, J. M. (2005). BINOL: a versatile chiral reagent. Chem. Rev. 105, 857-898. doi: $10.1021 / \mathrm{cr} 040079 \mathrm{~g}$

Brunel, J. M. (2007). Update 1 of: BINOL: a versatile chiral reagent. Chem. Rev. 107, PR1-PR45. doi: 10.1021/cr078004a

Christie, G. H., and Kenner, J. (1922). LXXI.-The molecular configurations of polynuclear aromatic compounds. Part I. The resolution of $\gamma-6: 6^{\prime}$-dinitro- and $4: 6: 4^{\prime}: 6^{\prime}$-tetranitro-diphenic acids into optically active components. J. Chem. Soc. Trans. 121, 614-620. doi: 10.1039/CT9222100614

Clayden, J., Moran, W. J., Edwards, P. J., LaPlante, S. R. (2009). The challenge of atropisomerism in drug discovery. Angew. Chem. Int. Ed. 48, 6398-6401. doi: $10.1002 /$ anie.200901719

Ding, K., Ishii, A., and Mikami, K. (1999). Super high throughput screening (SHTS) of chiral ligands and activators: asymmetric activation of chiral diolzinc catalysts by chiral nitrogen activators for the enantioselective addition of diethylzinc to aldehydes. Angew. Chem. Int. Ed. 38, 497-501.

Eichelbaum, M., and Gross, A. S. (1996). Stereochemical aspects of drug action and disposition. Adv. Drug Res. 28, 1-64.

Ema, T., Tanida, D., and Sakai, T. (2007). Versatile and practical macrocyclic reagent with multiple hydrogen-bonding sites for chiral discrimination in NMR. J. Am. Chem. Soc. 129, 10591-10596. doi: 10.1021/ja073476s

Frazer, R. R., Petit, M. A., and Saunders, J. K. (1971). Determination of enantiomeric purity by an optically active nuclear magnetic resonance shift reagent of wide applicability. J. Chem. Soc. Chem. Commun. 1450-1451. doi: $10.1039 / \mathrm{c} 29710001450$

Genet, J. P., Ayad, T., and Ratovelomanana-Vidal, V. (2014). Electron-deficient diphosphines: the impact of DIFLUORPHOS in asymmetric catalysis. Chem. Rev. 114, 2824-2880. doi: 10.1021/cr4003243

Ghosh, I., Zeng, H., and Kishi, Y. (2004). Application of chiral lanthanide shift reagents for assignment of absolute configuration of alcohols. Org. Lett. 6, 4715-4718. doi: 10.1021/ol048061f

Ghosn, M. W., and Wolf, C. (2009). Chiral amplification with a stereodynamic triaryl probe: assignment of the absolute configuration and enantiomeric excess of amino alcohols. J. Am. Chem. Soc. 131, 16360-16361. doi: 10.1021/ja907741v

Goering, H. L., Eikenberry, J. N., and Koermer, G. S. (1971). Tris[3(trifluoromethylhydroxymethylene)-d-camphorato] europium(III). Chiral shift
Provincial Natural Science Foundation of China (LY18B020011) and Foundation of Zhejiang Educational Committee (Y201430852).

\section{SUPPLEMENTARY MATERIAL}

The Supplementary Material for this article can be found online at: https://www.frontiersin.org/articles/10.3389/fchem. 2018.00300/full\#supplementary-material reagent for direct determination of enantiomeric compositions. J. Am. Chem. Soc. 93, 5913-5914. doi: 10.1021/ja00751a065

Gualandi, A., Grilli, S., Savoia, D., Kwit, M., and Gawronski, J. (2011). Chexaphenyl-substituted trianglamine as a chiral solvating agent for carboxylic acids. Org. Biomol. Chem. 9, 4234-4241. doi: 10.1039/c0ob01192d

Han, S. M. (1997). Direct enantiomeric separations by high performance liquid chromatography using cyclodextrins. Biomed. Chromatogr. 11, 259-271.

Huang, H., Bian, G., Zong, H., Wang, Y., Yang, S., Yue, H., et al. (2016). Chiral sensor for enantiodiscrimination of varied acids. Org. Lett. 18, 2524-2527. doi: 10.1021/acs.orglett.6b00088

Iwaniuk, D. P., and Wolf, C. (2010). A versatile and practical solvating agent for enantioselective recognition and NMR analysis of protected amines. J. Organ. Chem., 75, 6724-6727. doi: 10.1021/jo101426a

James, T. D., Sandanayake, R. A. S., and Shinkai, S. (1995). Chiral discrimination of monosaccharides using a fluorescent molecular sensor. Nature 374, 345-347. doi: $10.1038 / 374345 \mathrm{a} 0$

Kumobayashi, H., Miura, T., Sayo, N., Saito, T., and Zhang, X. (2001). Recent advances of BINAP chemistry in the industrial aspects. Synlett, 2001(Special Issue), 1055-1064. doi: 10.1055/s-2001-14625

Labuta, J., Ishihara, S., Šikorský, T., Futera, Z., Shundo, A., Hanyková, L., et al. (2013). NMR spectroscopic detection of chirality and enantiopurity in referenced systems without formation of diastereomers. Nat. Commun. 4:2188. doi: $10.1038 /$ ncomms3188

Lancelot, C. J., Harper, J. J., and von Schleyer, P. R. (1969). Participation by neighboring aryl groups. II. Accurate determinations of inductive and anchimeric assistance effects by a Hammett-Taft correlation. J. Am. Chem. Soc. 91, 4294-4296. doi: 10.1021/ja01043a051

Li, Z. B., Lin, J., and Pu, L. (2005). A cyclohexyl-1,2-diamine-derived bis(binaphthyl) macrocycle: enhanced sensitivity and enantioselectivity in the fluorescent recognition of mandelic acid. Angew. Chem. Int. Ed. 44, 1690-1693. doi: 10.1002/anie.200462471

Liu, C. X., Zheng, L., Zhu, L., Xiao, H. P., Li, X., and Jiang, J. (2017). Efficient chiral $1 \mathrm{H}$ NMR analysis of indoloquinazoline alkaloids phaitanthrin A, cephalanthrin-A and their analogues with a chiral phosphoric acid. Org. Biomol. Chem. 15, 4314-4319. doi: 10.1039/C7OB00823F

Liu, H.-L., Hou, X.-L., and Pu, L. (2009). Enantioselective precipitation and solidstate fluorescence enhancement in the recognition of $\alpha$-hydroxycarboxylic acids. Angew. Chem. Int. Ed. 48, 382-385. doi: 10.1002/anie.200804538

Lovely, A. E., and Wenzel, T. J. (2006). Chiral NMR discrimination of secondary amines using (18-Crown-6)-2,3,11,12-tetracarboxylic acid. Org. Lett. 8, 2823-2826. doi: 10.1021/ol0609558

Ma, Q., Ma, M., Tian, H., Ye, X., Xiao, H., Chen, L., et al. (2012). A novel amine receptor based on the binol scaffold functions as a highly effective chiral shift reagent for carboxylic acids. Org. Lett. 14, 5813-5815. doi: 10.1021/ol3027686

Mei, X., and Wolf, C. (2004). A highly congested N,N'-dioxide fluorosensor for enantioselective recognition of chiral hydrogen bond donors. Chem. Comm. 2004, 2078-2079. doi: 10.1039/B407718K

Miyashita, A., Yasuda, A., Takaya, H., Toriumi, K., Ito, T., Souchi, T., et al. (1980). Synthesis of 2,2'-bis(diphenylphosphino)-1,1'-binaphthyl (BINAP), an atropisomeric chiral bis(triaryl)phosphine, and its use in the rhodium(I)catalyzed asymmetric hydrogenation of alpha.-(acylamino)acrylic acids. J. Am. Chem. Soc. 102, 7932-7934. doi: 10.1021/ja00547a020 
Moon, L. S., Pal, M., Kasetti, Y., Bharatam, P. V., and Jolly, R. S. (2010). Chiral solvating agents for cyanohydrins and carboxylic acids. J. Org. Chem. 75, 5487-5498. doi: 10.1021/jo100445d

Nieto, S., Dragna, J. M., and Anslyn, E. V. (2010). A facile circular dichroism protocol for rapid determination of enantiomeric excess and concentration of chiral primary amines. Chem. Eur. J. 16, 227-232. doi: 10.1002/chem.200902650

Nieto, S., Lynch, V. M., Anslyn, E. V., Kim, H., and Chin, J. (2008). Highthroughput screening of identity, enantiomeric excess, and concentration using MLCT transitions in CD spectroscopy. J. Am. Chem. Soc. 130, 9232-9233. doi: $10.1021 /$ ja803443j

Parker, D. (1991). NMR determination of enantiomeric purity. Chem. Rev. 91, 1441-1457.

Pham, N. H., and Wenzel, T. J. (2011). Water-soluble calix[4]resorcinarene with $\alpha-$ methyl-1-prolinylmethyl groups as a chiral NMR solvating agent. J. Org. Chem. 76, 986-989. doi: 10.1021/jo102197w

Pirkle, W. H. (1966). The nonequivalence of physical properties of enantiomers in optically active solvents. differences in nuclear magnetic resonance spectra. I. J. Am. Chem. Soc. 88:1837. doi: 10.1021/ja00960a060

$\mathrm{Pu}, \mathrm{L}$. (2004). Fluorescence of organic molecules in chiral recognition. Chem. Rev. 104, 1687-1716. doi: 10.1021/cr030052h

Quinn, T. P., Atwood, P. D., Tanski, J. M., Moore, T. F., and Folmer-Andersen, J. F. (2011). Aza-crown macrocycles as chiral solvating agents for mandelic acid derivatives. J. Org. Chem. 76, 10020-10030. doi: 10.1021/jo2018203

Reetz, M. T., Becker, M. H., Kühling, K. M., and Holzwarth, A. (1998). Timeresolved IR-thermographic detection and screening of enantioselectivity in catalytic reactions. Angew. Chem. Int. Ed. 37, 2647-2650.

Reetz, M. T., Kühling, K. M., Deege, A., Hinrichs, H., and Belder, D. (2000). Superhigh-throughput screening of enantioselective catalysts by using capillary array electrophoresis. Angew. Chem. Int. Ed. 39, 3891-3893. doi: 10.1002/15213773(20001103)39:21<3891::AID-ANIE3891>3.0.CO;2-1

Schurig, V., and Nowotny, H. P. (1990). Gas chromatographic separation of enantiomers on cyclodextrin derivatives. Angew. Chem. Int. Ed. 29, 939-957. doi: 10.1002/anie.199009393

Seco, J. M., Quinoa, E., and Riguera, R. (2004). The assignment of absolute configuration by NMR. Chem. Rev. 104, 17-118. doi: 10.1021/cr000665j

Smyth, J. E., Butler, N. M., and Keller, P. A. (2015). A twist of naturethe significance of atropisomers in biological systems. Nat. Prod. Rep. 32, 1562-1583. doi: 10.1039/C4NP00121D
Tumambac, G. E., and Wolf, C. (2005). Enantioselective analysis of an asymmetric reaction using a chiral fluorosensor. Org. Lett. 7, 4045-4048. doi: $10.1021 / 010516216$

Wenzel, T. J. (2007). Discrimination of Chiral Compounds Using NMR. Hoboken, NJ: John Wiley \& Sons.

Wenzel, T. J., and Chisholm, C. D. (2011). Assignment of absolute configuration using chiral reagents and NMR spectroscopy. Chirality 23, 190-214. doi: 10.1002/chir.20889

Wenzel, T. J., and Wilcox, J. D. (2003). Chiral reagents for the determination of enantiomeric excess and absolute configuration using NMR spectroscopy. Chirality 15, 256-270. doi: 10.1002/chir. 10190

Yang, D., Li, X. Y., Fan, F., and Zhang, D.-W. (2005). Enantioselective recognition of carboxylates: a receptor derived from $\alpha$-aminoxy acids functions as a chiral shift reagent for carboxylic acids. J. Am. Chem. Soc. 127, 7996-7997. doi: $10.1021 /$ ja051072z

Yeh, H. J. C., Balani, S. K., Yagi, H., Greene, R. M. E., Sharma, N. D., Boyd, D. R., et al. (1986). Use of chiral lanthanide shift reagents in the determination of enantiomer composition and absolute configuration of epoxides and arene oxides. J. Org. Chem. 51, 5439-5443. doi: 10.1021/jo0037 $6 \mathrm{a} 080$

Zhao, J., Fyles, T. M., and James, T. D. (2004). Chiral binol-bisboronic acid as fluorescence sensor for sugar acids. Angew. Chem. Int. Ed. 43, 3461-3464 doi: 10.1002/anie. 200454033

Zhou, Y., Ye, H., and You, L. (2015). Reactivity-based dynamic covalent chemistry: reversible binding and chirality discrimination of monoalcohols. J. Org. Chem. 80, 2627-2633. doi: 10.1021/jo502801g

Conflict of Interest Statement: The authors declare that the research was conducted in the absence of any commercial or financial relationships that could be construed as a potential conflict of interest.

Copyright (c) $2018 \mathrm{Wu}, \mathrm{Liu}, \mathrm{Li}, \mathrm{Xiao}, \mathrm{Li}$ and Jiang. This is an open-access article distributed under the terms of the Creative Commons Attribution License (CC BY). The use, distribution or reproduction in other forums is permitted, provided the original author(s) and the copyright owner(s) are credited and that the original publication in this journal is cited, in accordance with accepted academic practice. No use, distribution or reproduction is permitted which does not comply with these terms. 